\title{
Arithmetic is Determinate
}

\author{
Zachary Goodsell
}

May 20, 2021

\begin{abstract}
Orthodoxy holds that there is always a determinate fact of the matter about every arithmetical claim. Little argument has been supplied in favour of orthodoxy, and work of Field, Warren and Waxman, and others suggests that the presumption in its favour is unjustified. This paper supports orthodoxy by establishing the determinacy of arithmetic in a well-motivated modal plural logic (Theorem 1). Recasting this result in higher-order logic (Theorem 13) reveals that even the nominalist who thinks that there are only finitely many things should think that there is some sense in which arithmetic is true and determinate.
\end{abstract}

Keywords: philosophy of mathematics; indeterminacy; modal logic; plural logic; higher-order logic.

\section{Introduction}

This paper presents an argument that there is always a determinate fact of the matter about any given arithmetical claim. Equivalently, that every arithmetical truth is determinately true. An arithmetical truth is the sort of truth that can be expressed by an arithmetical sentence: a sentence that contains only arithmetical and (first-order) logical vocabulary. The view defended entails, for instance, that it is determinate whether Peano arithmetic is consistent, whether every even number greater than two is the sum of two primes, whether there are infinitely many twin primes, and so on.

Arithmetic is typically thought of as a paradigmatic case of a subject matter free from indeterminacy. Work of Field (1998), Warren and Waxman (2020), and others has cast doubt on this consensus. The dialectical sitution, however, is confused by these authors' conflation of two positions that could both be called "arithmetical determinacy". As they see it, for arithmetic to be indeterminate is for there to be arithmetical sentences whose truth-values are somehow underspecified. But there 
is another interesting (and hitherto neglected) question in the vicinity, namely that of whether the arithmetical propositions themselves are indeterminate. On this conception, for arithmetic to be indeterminate is for the numbers themselves to have an indeterminate structure, independently of how we speak about them. The main results of this paper concern this second question. Section 2 makes the distinction more clearly, and shows that the linguistic conception of arithmetical determinacy cannot be adjudicated on without also resolving the question of whether arithmetical propositions are determinate.

On the conception of determinacy as a property of propositions, the determinacy operator is most appropriately formalised as a modal operator. With certain logical assumptions in place governing this modal operator and its interaction with plural reasoning, the determinacy of arithmetic can be derived. Section 3 puts forward and motivates the relevant assumptions, and Section 4 presents the formal argument for arithmetical determinacy in full detail.

The main argument for arithmetical determinacy requires the slightly awkward assumption that there are some things that satisfy the second-order Peano axioms, and that are also all determinately distinct from one another. Both of these assumptions can be eliminated by running the argument in a natural higher-order logic. It is shown in Section 5 that generalised quantifiers, including being zero in number, being one in number, and so on, determinately play the role of natural numbers, and that they are determinately distinct, eliminating the need for individuals playing the relevant roles.

\section{Linguistic Indeterminacy}

For Field, Warren and Waxman, and their opponents in the literature, ${ }^{1}$ the question of whether arithmetic is determinate is the question of whether every arithmetical sentence is either determinately true or determinately false. These theorists interpret the "determinately" in a sentence like "determinately, there are infinitely many twin primes" as covertly predicating determinate truth of the sentence "there are infinitely many twin primes" (despite the fact that the first sentence makes no explicit mention of any sentences). By contrast, "determinately, there are infinitely many twin primes" is here interpreted compositionally: it says that the proposition that there are infinitely many twin primes is determinately true. For arithmetic to be indeterminate in this sense is a defect in how the number themselves are arranged, rather than in what our sentences mean.

${ }^{1}$ Contributions to this literature include McGee 1997, Feferman et al. 1999, Martin 2001, Parsons 2007, Hamkins 2012, Hamkins 2015, and Button and Walsh 2016. 
Thus, there are two ways of understanding arithmetical determinacy, and both seem perfectly good. Once we acknowledge a propositional operator interpretation of "determinacy", we can ask whether that propositional operator applies to propositions such as that the sentence "there are infinitely many twin primes" is true. On the other hand, supposing that we understand what it would be for the truth-values of sentences to be indeterminate, we can just as well ask whether the arrangement of the numbers itself suffers from the same factual defectiveness.

To be clear, whereas Field and Warren and Waxman suggest that arithmetical sentences might be indeterminate in truth-value, the central argument of this paper is that the numbers themselves have a determinate arrangement. There is therefore no immediate contact between the present argument and the literature. However, there is a very important sense in which Field, Warren and Waxman, and especially their opponents, should be concerned with the present question of whether arithmetic is determinate.

These philosophers debate whether there are multiple admissible interpretations of arithmetical language that disagree on the truth-values of some sentences (admissibility of interpretation being a term-of-art that means something like: an interpretation that is not determinately incorrect). Call the view that admissible interpretations all agree on the truth-values of sentences the determinacy of arithmetical reference. It is common ground that admissible interpretations of arithmetical language form models of first-order Peano arithmetic (in the sense that an admissible interpretation of "is a natural number" and "is less than" together form a model of Peano arithmetic). Those who accept the determinacy of arithmetical reference endorse the stronger claim that on any admissible interpretation, the interpretation of "is a natural number" forms a minimal infinite well-ordering when ordered by the interpretation of "is less than". Since minimal infinite well-orderings are all isomorphic to one another, this would ensure that they agree on all sentences, as required. In this way, the debate over arithmetical determinacy has become a debate over whether there are admissible interpretations of "is a natural number" and "is less than" that do not form a minimal infinite well-ordering.

There are various ways that the proponent of determinacy can back up this constraint on admissible interpretations. One that seems especially promising is an appeal to reference magnetism (following a suggestion of Lewis (1984)). The obvious naturalness of being a minimal infinite well-ordering means that it would be very surprising if it was only an accident that we ended up talking about an instance of this structure with our arithmetical language. So, one might reasonably think, it should be possible to determinately speak about such a structure.

However: no matter how the constraint is justified, the determinacy of arithmetical reference is insufficient for establishing arithmetical determinacy, or even the determinate truth or falsity of every arithmetical sentence. For even granting 
that every arithmetical sentence means exactly one proposition determinately, that proposition might itself be an indeterminate one. For this reason, the relevance of the debate over the determinacy of arithmetical reference depends importantly on whether arithmetic is determinate in the present, non-linguistic, sense.

\section{The Logic of Determinacy}

This section and the next work towards the following result:

Theorem 1 (Rig). Where $\varphi$ schematically ranges over first-order arithmetical sentences: if the natural numbers are determinately distinct from one another and each axiom of second-order Peano arithmetic is determinately true, then either it is determinate that $\varphi$ or it is determinate that $\neg \varphi$.

This section concerns the logical principles and assumptions that are necessary for the proof (which are codified in the logic Rig), which is then carried out in Section 4. One point of controversy that I wish to sidestep is the assumption of classical logic. Many philosophers and mathematicians expect classical logic to fail, in one way or another, when indeterminacy is involved. This idea is too radical for my tastes, and positing failure of classical logic in mathematics is especially radical. Orthodox mathematics, which involves drawing out complicated logical consequences of simpler assumptions, contains an explicit commitment to classical logic, and so the denial of classical logic here would require the revision of mathematics as it is normally practised. Perhaps such revision will ultimately prove necessary, but the question of whether arithmetic might be indeterminate is an interesting one even on the presupposition that orthodox mathematics is in good standing. Here, then, we will assume classical logic to be correct. Field, as well as Warren and Waxman, are happy to make this assumption as well. For example, Field writes

[...] when I say that certain mathematical sentences might lack determinate truth value, I do not intend to suggest that we must abandon classical reasoning in connection with those sentences. (Field 1998, p. 295)

With classical logic in the background, we can begin to discuss the principles specific to determinacy. The least controversial principles of determinacy concern its propositional modal logic:

Factivity For all $p$, if it is determinate that $p$, then $p$.

$$
(\Delta \varphi) \rightarrow \varphi
$$

(Where " $\Delta$ " formalises the determinacy operator.) 
Closure For all $p$ and $q$, if it is determinate that if $p$ then $q$, and it is determinate that $p$, then it is determinate that $q$.

$$
\Delta(\varphi \rightarrow \psi) \rightarrow(\Delta \varphi \rightarrow \Delta \psi)
$$

These will be assumed without argument.

Things become more contentious when quantifiers and identity are introduced. However, once various confusions are cleared up, there is a strong case to be made for each of the required principles. One disputed principle is the Determinacy of Identity:

Determinacy of Identity For all $x$ and $y$, if $x=y$, then it is determinate that $x=y$.

$$
\forall x y(x=y \rightarrow \Delta(x=y))
$$

Famously, Evans (1978) proved the Determinacy of Identity by assuming the determinacy of self-identity and Leibniz' Law. Informally, the proof is as follows: if it is not determinate that $x=y$, then $x$ and $y$ differ in whether they are determinately equal to $y$, because $y$ is determinately identical to $y$ by the determinacy of self-identity. By Leibniz' Law, things that differ in any respect are not identical, so $x$ and $y$ are not identical. Since both of these premisses are as certain as any philosophical principle can be, Evans' proof is a convincing one.

Evans' proof crucially depends on "determinately" being regimented as a propositional operator rather than a predicate of sentences. Leibniz' Law says that identical things have the same properties, not that different names for the same person do. Even if "Anne is Anne" is determinately true and Anne is Beth, "Anne is Beth" might not be determinately true because it might be indeterminate who "Beth" refers to. This would of course be a case where "Anne is Beth" is indeterminate in which proposition it expresses, and not a case where the proposition that Anne is Beth is itself indeterminate.

One might also wonder whether there can be indeterminate cases of distinctness. Evans thought not; he argued from the determinacy of identity to the determinacy of distinctness via an assumption to the effect that indeterminacy is always had determinately: if it is indeterminate whether $p$, then it is determinate that it is indeterminate that $p$. Any two things that are not determinately not identical are not determinately not determinately identical by the determinacy of identity, and so are determinately identical by the assumption. But although the argument is valid, Evans makes no effort to motivate the assumption that indeterminacy is always had determinately, and it is not an obvious one. Since the assumption is not necessary for the following argument it will be eschewed. Accordingly, we will not take a stand here on whether there are indeterminate cases of distinctness.

Similar remarks apply to the following principle: 
Converse Barcan Formula If it is determinate that everything is some way, then everything is determinately that way.

$$
(\Delta \forall x \varphi) \rightarrow \forall x \Delta \varphi
$$

There is a compelling argument for the Converse Barcan Formula that relies on the determinate truth of each instance of Universal Instantiation. The argument is as follows (where $y$ is any variable that does not occur in $\varphi$ ):

1. $\Delta((\forall x \varphi) \rightarrow \varphi[y / x])$

Assumption

2. $(\Delta \forall x \varphi) \rightarrow \Delta \varphi[y / x]$

1, Closure

3. $(\Delta \forall x \varphi) \rightarrow \forall y(\Delta \varphi[y / x])$

2, Universal Generalisation

The assumption, $\Delta((\forall x \varphi) \rightarrow \varphi[y / x])$, is the result of putting a "determinately" before the Universal Instantiation instance $(\forall x \varphi) \rightarrow \varphi[y / x]$. On the face of it, the classical logician should expect the theorems of classical logic to expresss determinate truths, so this provides excellent reason for accepting Converse Barcan Formula.

The converse of the Converse Barcan Formula — the Barcan Formula—states that if everything is determinately some way then determinately everything is that way. There seems to be no analogous argument for the Barcan Formula, and accordingly it is on much shakier ground than its converse. Like the Determinacy of Distinctness, the Barcan Formula is a reasonable-seeming principle that will not be assumed here.

The interaction between determinacy and plural reasoning will be crucial to the argument for arithmetical determinacy. We will take plural quantification for granted; regimenting sentences like "there are some things that are all and only the bald people in this room" as the result of concatenating a quantifier-like expression called a plural quantifier with an open formula containing a free plural variable: "for some $X$, everything is one of the $X$ if and only if it is a bald person in this room." No stand will be taken on whether plural quantification is "set theory in sheep's clothing", as Quine (1970) would have it (although much more needs to be said for Quine's view to be plausible, given that it is inconsistent to say that for any things there is a set containing exactly them). What's important for the argument is that the relevant principles of plural reasoning hold, whether or not they amount to claims about set theory in the end.

The standard assumptions governing plural reasoning generally are those of Comprehension and Extensionality: 
Comprehension If anything is any given way, then there are some things that are all and only the things that are that way. ${ }^{2}$

$$
(\exists x \varphi) \rightarrow \exists Y \forall x(Y x \leftrightarrow \varphi)
$$

(We use capital letters for plural variables, and write " $Y x$ " for " $x$ is one of the $Y \mathrm{~s}^{\prime \prime}$ )

Extensionality If these things are all and only those things, then these things and those things are the same.

$$
\forall X Y(\forall z(X z \leftrightarrow Y z) \rightarrow X=Y)
$$

For example: since there is a set that is not a member of itself, there are some things that are all and only the sets that are not members of themselves.

The arguments given for the Determinacy of Identity and Converse Barcan Formula carry over immediately to establish the obvious analogues of these principles for plural reasoning. Things get slightly less clear when considering more complicated interactions of plural reasoning with determinacy. Suppose that it can be indeterminate which musician is a bandmate of which other. For example, suppose that at some point in 1955 it was indeterminate whether it was Sonny Rollins or John Coltrane who was in the same band as Miles Davis. In that case, we wish to conclude that it was indeterminate which people where bandmates of Davis'. That is, for any people, it was not determinate at that time that they were all and only the people in the same band as Davis. For this inference to be valid, we require pluralities to have their members determinately. Otherwise, there might be some people such that it is indeterminate whether Coltrane is one of them, and so for all that has been said it could be that those people determinately were all and only Davis' bandmates. That is, we require at least the following principle:

Persistence If something is one of some things, then it is determinately one of them.

$$
\forall X \forall y(X y \rightarrow \Delta X y)
$$

Persistence might be objected to by turning the previous reasoning on its head. Wasn't there a time where it was indeterminate whether Coltrane or Rollins was one of the members of the Miles Davis Quintet? So shouldn't we conclude that there is

\footnotetext{
${ }^{2}$ In the formal logic we will allow an "empty plurality"- -some things that are zero in number. Nothing substantive hangs on this.
} 
a plurality that has some indeterminate members, namely those people who were in the Miles Davis Quintet at that time?

This reasoning is tempting, but flawed. Those very people who the Miles Davis Quintet consisted of at that time were either Davis, Garland, Chambers, Jones, and Rollins, or the first four and Coltrane. There are no other options. And there can be no indeterminacy regarding whether Coltrane is among either lot of five people. There is absolutely nothing special about determinacy in this regard: clearly, who was a member of the Miles Davis Quintet changed over time, but being one of Davis, Garland, and so on is a property you have eternally, if at all. Similarly, who joined which band was contingent; but you are one of some people necessarily if you are one of them to begin with.

Persistence says that pluralities cannot lose members. Also crucial is the idea that they cannot gain members either. This idea is not obviously captured by the converse of Persistence:

Converse Persistence For any things, everything that is not one of them is determinately not one of them.

$$
\forall X \forall y(\neg X y \rightarrow \Delta \neg X y)
$$

Converse Persistence is missing something crucial in the absence of the Barcan Formula (for the first-order universal quantifier). For suppose the Barcan Formula fails in this way: everything is determinately not a unicorn, but it is indeterminate whether there is a unicorn. In a sense, it is indeterminate what things exist, but not because anything exists only indeterminately. Rather, there are ways the world could be for all that is determinately the case, and had the world been one of those ways there would have been more things, including a unicorn. Converse Persistence fails to entail the obvious truth that even in these cases, Davis, Garland, Chambers, Jones, and Coltrane would not have counted any unicorns among them. What we need instead is the principle of Inextensibility:

Inextensibility If any things are all determinately some way, then it is determinate that they are all that way. ${ }^{3}$

$$
\forall X(\forall y(X y \rightarrow \Delta \varphi) \rightarrow \Delta \forall y(X y \rightarrow \varphi))
$$

When indeterminate cases of distinctness are ruled out, Inextensibility entails Converse Persistence. If each of Davis, Garland, Chambers, Jones, and Coltrane is determinately distinct from Rollins, then by Inextensibility it is determinate that

${ }^{3}$ See Linnebo 2013, pp. 211-212. 
Rollins is not one of those people. But here we avoid presupposing the Determinacy of Distinctness. If, somehow, it were indeterminate whether Coltrane and Rollins were distinct, then it would surely also be indeterminate whether Rollins was one of Coltrane and the others. So for the sake of being ecumenical, we will also avoid the assumption of Converse Persistence.

It might seem like deniers of arithmetical determinacy have antecedent reason to reject Inextensibility. Some proponents of arithmetical indeterminacy draw parallels between determinacy and formal provability in some recursively axiomatisable theory such as Peano arithmetic; and provability in any sufficiently strong recursively axiomatisable theory has no property parallel to Inextensibility. For example, if Peano arithmetic is consistent, then you can prove of each number that it doesn't encode a proof of the inconsistency of Peano arithmetic, but there is no proof that no number encodes a proof of inconsistency. Thus, the proponent of arithmetical indeterminacy may think the following pair of sentences consistent:

(i) Each of the natural numbers determinately does not encode a proof of the inconsistency of Peano arithmetic.

(ii) It is indeterminate whether one of the natural numbers encodes a proof of the inconsistency of Peano arithmetic.

And Inextensibility seems to rule out this situation from the get-go. In fact, this is not the case. The sentence (ii) contains a scope ambiguity that prevents conflict with (i). It can be disambiguated as follows:

(ii.a) It is indeterminate whether there is a natural number that encodes a proof of the inconsistency of Peano arithmetic.

(ii.b) Those very things that are the natural numbers are such that it is indeterminate whether one of them encodes a proof of the inconsistency of Peano arithmetic.

Inextensibility only says that (i) contradicts (ii.b). But it is (ii.a) that states that arithmetic is indeterminate, because (ii.a) is made from a sentence stating the inconsistency of Peano arithmetic prepended with an "it is indeterminate whether" operator. And (ii.a) is consistent with Inextensibility: if it is indeterminate which things are all and only the natural numbers; which is to say if it is not determinate for any things that they are all and only the natural numbers, then for all Inextensibilitys says it could be that while the things that happen to be the natural numbers are all determinately some way, it is not determinate that all natural numbers are that way. And for all that has been said so far, it could be indeterminate which things are all and only the natural numbers. Presumably, this is exactly the sort of thing that 
the proponent of arithmetical indeterminacy should argue for, and it is what will be argued against in what follows.

A technical extension of plural reasoning is the final logical component of the argument. This is the extension of plural reasoning and all of its interactions with the logic of determinacy to binary plural reasoning. What we want is a quantifier-like expression, "some pairs of things", that stands to binary relations just as the plural quantifier "some things" stands to properties. That is, we require a comprehension axiom along the lines of: for any way of relating things to things, if at least one pair of things is related in that way, there are some pairs of things that are all and only the pairs of things that are related in that way. Similarly, analogues of Extensionality, Persistence, and Inextensibility must hold for the argument to work. The cogency of this sort of quantification, and the plausibility of these principles, can be confirmed by beginning to reason with them. One will quickly realise that binary plural quantification makes just as much sense as unary plural quantification does, and that the relevant principles are just as necessary. Those who are still sceptical will be able to achieve the same effect by coding pluralities of pairs of natural numbers as unary pluralities, for example by treating the product of the $m^{\text {th }}$ and $n^{\text {th }}$ prime numbers as a proxy for the pair of numbers $(m, n){ }^{4}$

These principles form the core of the argument for arithmetical determinacy. Obviously some assumptions must be made about the natural numbers too. For all that has been said so far, the only natural numbers are certain borderline shades of blue-green, and to be less than a number is to be green. In that case it might be indeterminate whether any number is less than any other, but we of course know better. Here, it will be assumed that the natural numbers have an ordering, pronounced "is less than", that determinately obeys the following principles:

Ordering Being less than is a transitive, reflexive, and antisymmetric relation.

Zero There is a least natural number, 0 .

Successors Every natural number has a successor-a least natural number that it is strictly less than.

${ }^{4}$ Securing the binary plural analogues of Comprehension, Extensionality, Persistence and Inextensibility for this coded binary plural quantification will require the determinate truth of second-order Peano arithmetic, as well as assumptions to the effect that prime numbers are determinately prime, composite numbers are determinately composite, and the product of two numbers is determinately their product. These assumptions, I think, are uncontroversial, but if they are disputed we may fall back on taking binary plural quantification as primitive, and argue for the relevant principles of binary plural quantification directly in exactly the same way we established the analogous unary plural principles. 
Induction If 0 is one of some natural numbers, and the successor of every natural number among those numbers is also one of them, then every natural number is one of them.

If you don't think the natural numbers have these properties at all, then we may as well not bother talking about them and the whole question of arithmetical determinacy is moot. That these things hold determinately is less obvious. One argument in favour relies on a purported connection between determinacy and assertion: it is inappropriate to go around asserting indeterminate truths; but it is completely appropriate to assert that the natural numbers satisfy Ordering, Zero, and so on; so these properties are had determinately. In any case, deniers of arithmetical determinacy in the literature seem to have little interest in denying the determinacy of these basic principles, so it is safe to assume them. We will also require the determinate distinctness of natural numbers:

Determinately Distinct Numbers Distinct natural numbers are determinately distinct.

Nobody to my knowledge has doubted this principle in print. It would be very strange if it failed. If numbers $m$ and $m+n$ were only indeterminately distinct, what would happen if we put exactly $m$ apples and $n$ oranges together in a basket? Would it be somehow fuzzy or objectively unclear whether the basket has more fruits in total than it has apples? Determinately Distinct Numbers does not follow from the other principles, so it must be left as an assumption here.

\section{Deriving Arithmetical Determinacy}

With these principles in hand we can now establish the determinacy of any truth that can be stated with an arithmetical sentence. The proof breaks down into five components which can be stated informally:

1. If any things ordered in any way satisfy the principles Ordering, Zero, Successors, and Induction, then there is an order-preserving one-to-one correspondance (an isomorphism) between those things and the natural numbers (this is a famous result of Dedekind 1893). Thus, we can keep track of the structure of the natural numbers by keeping track of the inductive structures, which are pairs of a plurality of things and a plurality of pairs that together satisfy Ordering, Zero, Successors, and Induction. (Theorem 2)

2. If there is any inductive structure with determinately distinct members, then it is determinately an inductive structure. Thus, whichever things are all and 
only the natural numbers, alongside whichever pairs of things are all and only the pairs of natural numbers with the first less than the second, determinately form an inductive structure. (Lemma 4)

3. Whether a given arithmetical claim is true depends only on the structure of the natural numbers. More precisely: whether the chosen claim is true depends only on which things are all and only the natural numbers, and which pairs of things are all and only the pairs of natural numbers with the first less than the second. (Lemma 3)

4. By (1) and (2), the natural numbers with their ordering are determinately isomorphic to those things that are all and only the natural numbers, ordered by those pairs that are all and only those pairs of natural numbers with the first less than the second. In other words, the structure of the natural numbes is had determinately.

5. By (3) and (4), the given arithmetical claim is either determinately true or determinately false.

Formally, we work in a language $\mathcal{L}^{\Delta}$ with:

- Brackets denoting the order of operations.

- The standard Boolean truth-functional connectives: $\neg, \rightarrow, \wedge, \vee, \leftrightarrow$. We adopt the convention of associating strings of $\rightarrow$ s to the right: $\phi \rightarrow \chi \rightarrow \psi$ is an abbreviation for $\phi \rightarrow(\chi \rightarrow \psi)$.

- Typed variables, of types $e,\langle e\rangle$, and $\langle e, e\rangle . \quad e$ is syntactic type of names of numbers, $\langle e\rangle$ and $\langle e, e\rangle$ of plural variables and binary plural variables respectively. Where ambiguity is possible, a variable's type will be denoted with superscript, as in $X^{\langle e, e\rangle}$.

- Universal quantifiers over each type, always denoted with $\forall x$ where $x$ is a variable of the type quantified over. The existential quantifier $\exists x$ is an abbreviation for $\neg \forall x \neg$. We also use $\forall x y$ to abbreviate $\forall x \forall y$, when $x$ and $y$ have the same type.

- An equality connective =, which is used ambiguously to denote equality between individuals, between unary pluralities, and between binary pluralities.

- A sentential operator $\Delta$, to be interpreted as it is determinate that. 


\begin{tabular}{|ll|}
\hline PC & Theorems of classical propositional logic \\
UI & $(\forall x \varphi) \rightarrow \varphi[x / a]$ \\
LL & $x=y \rightarrow \varphi \rightarrow \varphi[x / y]$ \\
Ext1 & $\forall X Y(\forall z(X z \leftrightarrow Y z) \rightarrow X=Y)$ \\
Ext2 & $\forall X Y\left(\forall z z^{\prime}\left(X z z^{\prime} \leftrightarrow Y z z^{\prime}\right) \rightarrow X=Y\right)$ \\
Comp1 & $\exists X \forall y(X y \leftrightarrow \varphi)$, where $X$ is not free in $\varphi$ \\
Comp2 & $\exists X \forall y z(X y z \leftrightarrow \varphi)$, where $X$ is not free in $\varphi$ \\
K & $\Delta(\varphi \rightarrow \psi) \rightarrow \Delta \varphi \rightarrow \Delta \psi$ \\
T & $\Delta \varphi \rightarrow \varphi$ \\
Per1 & $\forall X \forall y(X y \rightarrow \Delta X y)$ \\
Per2 & $\forall X \forall y z(X y z \rightarrow \Delta X y z)$ \\
Inex1 & $\forall X(\forall y(X y \rightarrow \Delta \varphi) \rightarrow \Delta \forall y(X y \rightarrow \varphi))$ \\
Inex2 & $\forall X(\forall y z(X y z \rightarrow \Delta \varphi) \rightarrow \Delta \forall y z(X y z \rightarrow \varphi))$ \\
\hline MP & If $\vdash \varphi$ and $\vdash \varphi \rightarrow \psi$, then $\vdash \psi$ \\
Gen & If $\vdash \varphi \rightarrow \psi$ where $x$ (of any type $)$ is not free in $\varphi$, then $\vdash \varphi \rightarrow \forall x \psi$ \\
Nec & If $\vdash \varphi$ then $\vdash \Delta \varphi$ \\
\hline
\end{tabular}

Table 1: Axioms and rules of Rig.

Formulae are formed recursively. The atomic formulae are equalities $x=y$ for $x$ and $y$ of any one type, as well as applications $X y$ and $W y z$ where $y$ and $z$ are of type $e, X$ of type $\langle e\rangle$, and $W$ of type $\langle e, e\rangle$. Intuitively, $X y$ states that $y$ is one of the $X$, and $W y z$ that the pair $(y, z)$ is one of the $W$. The rest of the formulae are then generated by Boolean combinations of formulae and the prepending of $\Delta$ and quantifiers. The axioms and rules of our formal system Rig are listed in Table 1.

To begin with we require three definitions.

Definition 1 (Total ordering). Some pairs of things $\leq$ totally order some things $X$ if $\leq$ is reflexive, connexive, transitive, and antisymmetric when restricted to $X$. In symbols:

$$
\begin{aligned}
\operatorname{Order}\left(X^{\langle e\rangle}, \leq^{\langle e, e\rangle}\right): & \forall x y((X x \wedge X y) \rightarrow \\
& x \leq x \\
& \wedge(x \leq y \vee y \leq x) \\
& \wedge((x \leq y \wedge y \leq z) \rightarrow x \leq z) \\
& \wedge((x \leq y \wedge y \leq x) \rightarrow x=y)) .
\end{aligned}
$$

Definition 2 (Inductive structure). Some things $X$ and some total ordering of them $\leq$ form an inductive structure (and we say Ind $X \leq$ ) just in case (a) there is a $\leq$-minimal $X$ thing, (b), every $X$ thing has a unique $\leq$-successor, and (c), if the $\leq$-minimal thing 
is one of some things that always includes the successor of any $X$ thing that is one of them, then every $X$ thing is one of them. Formally:

$$
\begin{aligned}
\operatorname{Ind}\left(X^{\langle e\rangle}, \leq^{\langle e, e\rangle}\right) & := \\
\exists 0(X 0 & \wedge \text { Order } X \leq \\
& \wedge \forall x(X x \rightarrow 0 \leq x) \\
& \wedge \forall x\left(X x \rightarrow \exists y\left(X y \wedge \operatorname{Suc}_{X \leq} x y\right)\right) \\
& \left.\wedge \forall Y^{\langle e\rangle}(Y 0 \wedge \forall y(Y y \rightarrow \forall z(\operatorname{Suc} y z \rightarrow Y z)) \rightarrow \forall y(X y \rightarrow Y y))\right) .
\end{aligned}
$$

(where 'Suc $X \leq x y$ ' abbreviates ' $X x \wedge X y \wedge x<y \wedge \forall z(x<z \rightarrow y \leq z)$ ', and the subscript $X \leq$ is omitted where obvious.)

Definition 3 (Isomorphism/Isomorphic). Some pairs of things $\iota$ form an isomorphism between $S^{\langle e\rangle}, R^{\langle e, e\rangle}$ and $S^{\prime\langle e\rangle}, R^{\prime\langle e, e\rangle}$ if $\iota$ bijectively relates the $S$ things and the $S^{\prime}$ things so that the $R$ pairs (i.e., " $R$-related things") are mapped to the $R^{\prime}$ pairs, and vice-versa. That is,

$$
\begin{aligned}
\operatorname{Iso}\left(S, R, S^{\prime}, R^{\prime}, \iota\right) & :=\forall x y\left(\iota x y \rightarrow S x \wedge S^{\prime} y\right) \\
& \wedge \forall x\left(S x \rightarrow \exists y\left(S^{\prime} y \wedge \iota x y\right)\right) \\
& \wedge \forall y\left(S^{\prime} y \rightarrow \exists x(S x \wedge \iota x y)\right) \\
& \wedge \forall x y z(\iota x y \rightarrow \iota x z \rightarrow y=z) \\
& \wedge \forall x y z(\iota x y \rightarrow \iota z y \rightarrow x=z) \\
& \wedge \forall w x y z(\iota w y \wedge \iota x z \rightarrow(R w x \leftrightarrow R y z))
\end{aligned}
$$

Say $S, R$ and $S^{\prime}, R^{\prime}$ are isomorphic if there is an isomorphism between them.

Next up is Dedekind's (1893) categoricity theorem carried out in Rig.

Theorem 2 (Rig). Any two inductive structures are isomorphic.

Proof. Take inductive structures consisting respectively of $S$ and $R$ and $S^{\prime}$ and $R^{\prime}$. Let $\kappa$ be those pairs of things coextensive with the condition being an $S$ thing and an $S^{\prime}$ thing respectively whose predecessors are isomorphic. The existence of $\kappa$ follows from Comp; I will now show that $\kappa$ is an isomorphism between $S$ and $S^{\prime}$.

To show that $\kappa$ is a bijection, use induction: 0 and $0^{\prime}$ - the $R$-minimal and $R^{\prime}$ minimal things respectively - are such that there is a isomorphism between things less than them, namely the empty plurality of pairs. For the inductive step, suppose that $\iota$ is an isomorphism between the things less than $x$ and the things less than $x^{\prime}$, then by Comp let $\iota^{+}$be coextensive with $\iota y z \vee\left(y=x \wedge z=x^{\prime}\right) . \iota^{+}$is then an isomorphism between the things less than the successors of $x$ and $x^{\prime}$ respectively. Isomorphicness is obviously transitive, so by double induction, $\kappa$ is a bijection. 
To show that $\kappa$ maps $R$-related things to $R^{\prime}$-related things, use induction again. The base case (i.e., if $\kappa x x^{\prime}$ then if $0 \leq x$ if and only if $0^{\prime} \leq x^{\prime}$ ) is again trivial, and the inductive step follows immediately from the above observation that $\kappa$ preserves successorhood in both directions.

Next up is to show that the way some pairs of things $R$ relate some things $S$ is never indeterminate, if the $S$ things are determinately distinct. To state this idea precisely, we require the following definition:

Definition 4 ( $S R$-first-order formula/sentence). The class of $S R$-first-order formulae is defined recursively as follows:

- $x=y$ and $R x y$ (where $x$ and $y$ are any variables of type $e$ ) are $S R$-first-order formulae.

- If $\varphi$ and $\psi$ are $S R$-first-order formulae, then so are $\neg \varphi, \varphi \wedge \psi, \varphi \vee \psi, \varphi \rightarrow \psi$, and $\forall x(S x \rightarrow \varphi)$, where $x$ is any variable of type $e$.

An $S R$-first-order sentence is an $S R$-first-order formulae whose only free variables are $S$ and $R$.

Notice that replacing ' $S$ ' and ' $R$ ' in the definition with predicates for being a natural number and being less than would yield an adequate definition of the first-order arithmetical formulae.

We will now show that when the $S$ things are determinately distinct from one another, every $S R$-first-order sentence expresses something determinate. As remarked in Section 3, this is not surprising: indeterminacy should arise, if at all, from it being indeterminate which things the natural numbers are, and which pairs are coextensive with the natural number ordering.

Lemma 3 (Rig). Where Dist $S$ abbreviates $\forall x y(S x \rightarrow S y \rightarrow x \neq y \rightarrow \Delta x \neq y)$,

$$
\text { Dist } S \rightarrow \forall x_{1} \ldots x_{n}\left(S x_{1} \rightarrow \cdots \rightarrow S x_{n} \rightarrow(\Delta \varphi \vee \Delta \neg \varphi)\right),
$$

where $\varphi$ schematically ranges over SR-first-order formulae with at most $x_{1}$ through $x_{n}$ free.

Proof. By induction on the complexity of $\varphi$. The base cases are when $\varphi$ is of the form $x=y$ or $R x y$. In the first case we use either the determinacy of identity or Dist $S$; in the second we use either Inex and Dist $S$ or Per.

Now suppose that $\varphi$ is a Boolean combination of formulae for which the result holds. It is easy to check that a Boolean combination of either determinately true or determinately false things is either determinately true or determinately false, so the result will hold for $\varphi$ too. 
If $\varphi$ is of the form $\forall x(S x \rightarrow \psi)$ with exactly $x_{1}$ through $x_{n}$ (distinct from $x$ ) free, and we have proved the result for $\psi$, then on the assumption that $S x_{1} \wedge \cdots \wedge S x_{n}$ we have $\forall x(S x \rightarrow(\Delta \psi \vee \Delta \neg \psi))$ by hypothesis. Any counterexample to $\forall x(S x \rightarrow \psi)$ is therefore a determinate counterexample, because if $S x \wedge \neg \psi$ holds then so does $\Delta S x \wedge \Delta \neg \psi$ by Per and the inductive hypothesis. Modal logic then yields $\Delta(S x \wedge \neg \psi)$. By Converse Barcan Formula, then, if there is a counterexample to $\varphi$ then $\Delta \neg \varphi$ holds.

On the other hand, if there is no counterexample, then we have $\forall x(S x \rightarrow \Delta \psi)$ by the inductive hypothesis, which by Inex applied to $S$ entails $\Delta \forall x(S x \rightarrow \psi)$, which is $\Delta \varphi$. Discharging the assumption that $S x_{1} \wedge \cdots \wedge S x_{n}$ and that Dist $S$ gives

$$
\text { Dist } S \rightarrow \forall x_{1} \ldots x_{n}\left(S x_{1} \rightarrow \cdots \rightarrow S x_{n} \rightarrow(\Delta \varphi \vee \Delta \neg \varphi)\right)
$$

as required.

Lemma 4 (Rig). Dist $S \rightarrow \operatorname{Ind}(S, R) \rightarrow \Delta \operatorname{Ind}(S, R)$.

Proof. The first three components of $S$ and $R$ forming an inductive structure-that $R$ totally orders $S$; that there is an $R$-minimal $S$ thing; and that every $S$ thing has an $R$-successor-are all statable by $S R$-first-order formulae. Thus, they all hold determinately of $S$ and $R$, if at all, by Lemma 3 .

The final component of being a determinately inductive structure is satisfying the induction principle determinately:

$$
\Delta \forall n\left(\operatorname{Sn} \rightarrow \forall X^{\langle e\rangle}(X 0 \wedge \forall x(X x \rightarrow \forall y(\operatorname{Suc} x y \rightarrow X y)) \rightarrow X n)\right) .
$$

By Inex applied to $S$, this is entailed by

$$
\forall n\left(S n \rightarrow \Delta \forall X^{\langle e\rangle}(X 0 \rightarrow \forall x(X x \rightarrow \forall y(\operatorname{Suc} x y \rightarrow X y)) \rightarrow X n)\right) .
$$

Informally, this is the claim that every $S$ thing is determinately such that it can be reached from the first $S$ thing by a finite chain of successors. This will be established by induction.

The base case is trivial. For the inductive step, notice that the following is a theorem of Rig:

$$
\text { Suc } m n \rightarrow \forall X(X 0 \rightarrow \forall x(X x \rightarrow \forall y(\operatorname{Suc} x y \rightarrow X y)) \rightarrow X m \rightarrow X n)
$$

Necessitating, and then distributing the $\Delta$ over the conditional, we get

$$
\Delta \operatorname{Suc} m n \rightarrow \Delta \forall X(X 0 \rightarrow \forall x(X x \rightarrow \forall y(\operatorname{Suc} x y \rightarrow X y)) \rightarrow X m \rightarrow X n)
$$

Supposing for induction that the claim holds for an arbitary $m$, we get

$$
\Delta \operatorname{Suc} m n \rightarrow \Delta \forall X(X 0 \rightarrow \forall x(X x \rightarrow \forall y(\operatorname{Suc} x y \rightarrow X y)) \rightarrow X n)
$$

So suppose Suc $m n$. Then $\Delta$ Suc $m n$ by Lemma 3, so $n$ has the required property. 
Theorem 5 (Rig).

$$
\Delta(\exists S R(\text { Ind } S R \wedge \text { Dist } S) \rightarrow(\Delta \forall S R(\text { Ind } S R \rightarrow \varphi) \vee \Delta \forall S R(\text { Ind } S R \rightarrow \neg \varphi))),
$$

where $\varphi$ schematically ranges over $S R$-first-order formulae.

Proof. Assume Ind $S R$ and Dist $S$. Then $\triangle$ Ind $S R$ (Lemma 4); so determinately if Ind $S^{\prime} R^{\prime}$ then $S$ and $S^{\prime}$ are isomorphic (Theorem 2). By Lemma 3, this entails

$$
\Delta \forall S^{\prime} R^{\prime}\left(\text { Ind } S^{\prime} R^{\prime} \rightarrow \varphi \rightarrow \varphi\left(S^{\prime}, R^{\prime}\right)\right. \text { ), }
$$

where $\varphi\left(S^{\prime}, R^{\prime}\right)$ is the result of replacing every $S$ in $\varphi$ with an $S^{\prime}$ and every $R$ with an $R^{\prime}$. Also by Lemma 3, $\varphi$ is determinately true if it is true. Since $S^{\prime}$ and $R^{\prime}$ are not free in $\varphi$, we can conclude

$$
\varphi \rightarrow \Delta \forall S^{\prime} R^{\prime}\left(\text { Ind } S^{\prime} R^{\prime} \rightarrow \varphi\left(S^{\prime}, R^{\prime}\right)\right)
$$

Since all the same would clearly go for $\neg \varphi$, it follows that

$$
\Delta \forall S^{\prime} R^{\prime}\left(\operatorname{Ind} S^{\prime} R^{\prime} \rightarrow \varphi\left(S^{\prime}, R^{\prime}\right)\right) \vee \Delta \forall S^{\prime} R^{\prime}\left(\operatorname{Ind} S^{\prime} R^{\prime} \rightarrow \neg \varphi\left(S^{\prime}, R^{\prime}\right)\right)
$$

which is modulo a change of variables the very same claim as

$$
\Delta \forall S R(\text { Ind } S R \rightarrow \varphi) \vee \Delta \forall S R(\text { Ind } S R \rightarrow \neg \varphi) \text { ). }
$$

To reach this conclusion we assumed Ind $S R$ and Dist $S$ for an arbitrary $S$ and $R$, so we can prove with no assumptions that

$$
\exists S R(\text { Ind } S R \wedge \text { Dist } S) \rightarrow(\Delta \forall S R(\text { Ind } S R \rightarrow \varphi) \vee \Delta \forall S R(\text { Ind } S R \rightarrow \neg \varphi)) .
$$

The result is then given by the rule Nec.

Theorem 1 (Rig). Where $\varphi$ schematically ranges over first-order arithmetical sentences: if the natural numbers are determinately distinct from one another and each axiom of second-order Peano arithmetic is determinately true, then either it is determinate that $\varphi$ or it is determinate that $\neg \varphi$.

Proof. Immediate from Theorem 5 once the language $\mathcal{L}^{\Delta}$ is augmented with predicates for being a natural number and is less than.

This completes the argument for the determinacy of arithmetic. An interesting question is whether a similar argument carries through to establish the determinacy of mathematics more generally, for instance by yielding the determinacy of analysis or set theory. It turns out that the situation is much more complex. Whereas various reasonable-seeming additions to Rig suffice for the determinacy of set theory, Rig alone does not, even when augmented with generous assumptions to the effect that membership is always had determinately if it all, and that the sets determinately satisfy the second-order ZFC axioms. ${ }^{5}$ Theorem 1 therefore constitutes only the beginning of the investigation of mathematical indeterminacy more generally.

${ }^{5}$ Proof of this claim is in unpublished work. 


\section{Generalising the Determinacy Theorem}

\begin{tabular}{|c|c|}
\hline $\mathrm{PC}$ & Theorems of classical propositional logic \\
\hline UI & $\left(\forall_{\sigma} x \varphi\right) \rightarrow \varphi[x / a]$ \\
\hline LL & $x=y \rightarrow \varphi \rightarrow \varphi[x / y]$ \\
\hline$\beta \eta$ & $\varphi \leftrightarrow \psi$, when $\varphi$ and $\psi$ are $\beta \eta$-equivalent \\
\hline $\mathrm{Eq}$ & $\varphi=\psi$ and $\lambda \bar{z} . \phi=\lambda \bar{z} . \psi$, when $\mathrm{H} \vdash \varphi \leftrightarrow \psi$ \\
\hline \multirow[t]{2}{*}{ Rigidity } & $\forall X^{\sigma_{1} \rightarrow \cdots \rightarrow \sigma_{n} \rightarrow t} \exists Y($ Rigid $Y \wedge \forall \bar{z}(X \bar{z} \leftrightarrow Y \bar{z}))$, where \\
\hline & Rigid $:=\lambda X^{\sigma_{1} \rightarrow \cdots \rightarrow \sigma_{n} \rightarrow t} . \square \forall Y(\forall \bar{z}(X \bar{z} \rightarrow \square Y \bar{z}) \leftrightarrow \square \forall \bar{z}(X \bar{z} \rightarrow Y \bar{z}))$ \\
\hline MP & If $\vdash \varphi$ and $\vdash \varphi \rightarrow \psi$, then $\vdash \psi$ \\
\hline Gen & If $\vdash \varphi \rightarrow \psi$ and $x^{\sigma}$ is not free in $\varphi$, then $\vdash \varphi \rightarrow \forall x \psi$ \\
\hline
\end{tabular}

Table 2: $\mathrm{H}$ is the logic whose axioms are PC, UI, LL, and with the rules MP and Gen. C, or Classicism, extends $\mathrm{H}$ by the axiom schema Eq. C + Rigidity extends Classicism by the axiom schema of Rigidity.

The conclusion of Theorem 1 can be avoided by denying that the natural numbers have their expected properties determinately. However, Theorem 5 establishes a result that is almost as good: that it is determinate which sentences of arithmetic inductive structures satisfy, when considered as models of arithmetic.

Theorem 5 assumes that there is an inductive structure whose members are determinately distinct. Nominalists who deny the existence of an inductive structure, as well as those who expect distinctness facts to be modally fragile, have reason to deny the philosophical relevance of Theorem 5. Frege 1884 suggested that the quantifiers themselves can, in some sense, play the role of natural numbers. Following through with this suggestion in a natural higher-order logic will allow us to explicitly define and prove the existence of a witness to the existential antecedent of Theorem 5, thereby defusing both worries.

Let us now switch languages to the relational simply typed $\lambda$-calculus, which has types defined recursively starting from base types $e$ and $t$ and combining types $\sigma$ and $\tau$, with $\tau \neq e$, into the higher type $\sigma \rightarrow \tau$. The concatenation of a term of type $\sigma \rightarrow \tau$ to a term of type $\sigma$ is a term of type $\tau$, and free variables of type $\sigma$ in a term of type $\tau$ are bound by $\lambda$-abstraction to create terms of type $\sigma \rightarrow \tau$. $e$ is again the type of names, now $t$ is the type of sentences, $e \rightarrow t$ of unary predicates, and so on. The constants of the language are the truth-functional connectives as well as universal quantifiers and identity for each type. Call this language $\mathcal{H}$.

Our logic of choice is Classicism with the additional axiom schema of Rigidity. Both Classicism and Rigidity are first isolated by Bacon and Dorr (forthcoming). The axioms and rules of this logic are collated in Table 2; in the following paragraphs I will describe the logic informally. Classicism is an extension of the logic $\mathrm{H}$, which 
is introduced in Bacon 2018 ( $\mathrm{H}$ is a weakening of Church's (1940) simple theory of types). $\mathrm{H}$ is the minimal logic in which

- The material conditional $\rightarrow$ behaves in the classical way ( $\perp$ is defined as $\forall_{t} p(p)$, negation as $\lambda p .(p \rightarrow \perp)$, and so on for the other truth-functional connectives).

- Universal quantifers of all types obey analogues of universal instantiation and universal generalisation. So, for instance, you can infer $F a$ from $\forall X(X a)$.

- $\beta \eta$-equivalent expressions are intersubstitutable. This amounts to the following two claims about how $\lambda$-expressions behave:

- When $x$ is a type $\sigma$ variable and $M$ an arbitrary expression of type $\sigma \rightarrow \tau$ with $x$ not free, $\lambda x . M x$ is intersubstitable with $M$.

- Where $x$ is any variable and $a$ is an arbitrary expression of the same type, $(\lambda x . M) a$ is intersubstitutable with $M[a / x]$, when $a$ does not contain any variables that become bound when substituting $a$ for $x$ in $M$.

In $\mathcal{H}$, one can define a notion of identity for each type,${ }_{\sigma}$ :

$$
\left(=_{\sigma}\right):=\lambda x^{\sigma} y . \forall Z(Z x \rightarrow Z y)
$$

Classicism extends $\mathrm{H}$ with some claims about how these identity connectives behave. For all $\mathrm{H}$ says, conjunction is not symmetric, in the sense that $\lambda p q .(p \wedge q)$ might not be equal to $\lambda p q .(q \wedge p)$. Classicism rules out this sort of situation by upgrading all provable equivalences to identities. So, for instance, since $(p \wedge q) \leftrightarrow(q \wedge p)$ is a theorem of the propositional calculus, Classicism is to include the identity $\lambda p q \cdot(p \wedge q)=\lambda p q \cdot(q \wedge p)$. This effect can be achieved by the addition of the axiom schema Eq. The instances of Eq are identities of the form $\varphi=\psi$ and $\left(\lambda x_{1} \ldots x_{n} . \varphi\right)=_{\sigma_{1} \rightarrow \cdots \rightarrow \sigma_{n} \rightarrow t}\left(\lambda x_{1} \ldots x_{n} . \psi\right)$, where $\varphi \leftrightarrow \psi$ is a theorem of $\mathrm{H}$.

The effect of including Eq is that sentences that are provable in Classicism all state the same thing (this is a result of Bacon and Dorr). In particuar, if you can prove $\varphi$ in Classicism, you can also prove $\varphi=T$, where ' $T$ ' is your favourite tautology (e.g., $\forall p .(p) \rightarrow \forall p .(p))$. From this we can show that being identical to $\mathrm{T}$, $\lambda p .(p=T)$, has a Quantified KT modal logic, just like determinacy and other notions of necessity. Thus, it is helpful to think of $\lambda p .(p=T)$ as a notion of necessity itself. We will denote it with ' $\square$ ' and pronounce it as necessarily. Clearly, everything that is necessary in this extremely demanding sense is determinate, because there is only one thing that is necessary, $T$, and $T$ is a determinate truth. Another important but not immediately obvious consequence of Classicism, also established by Bacon 
and Dorr, is that necessarily coextensive properties, propositions, and relations are identical.

A commitment to Classicism is implicit in orthodox treatments of propositions as sets of possible worlds and properties as functions from individuals to sets of possible worlds. ${ }^{6}$ Of course, the tenets of Classicism remain uncertain, and no argument can be made decisively in their favour here, but Classicism's status as an orthodox starting point should not be controversial.

For the present argument to work, Classicism must be extended by the axiom schema of Rigidity. One instance of the schema says, roughly, that every property is coextensive with a rigid property, which we define as a property satisfying analogues of Extensionality, Persistence, and Inextensibility with necessity replacing determinacy (the official definition, stated in Table 2, collates these three properties into a single condition; proving that they hold is an instructive exercise). The other instances say the same thing but for higher-order properties and relations of all types. Rigidity is motivated by an appeal to plural reasoning, just as the original Peristence and Inextensibility principles were motivated in Section 3. The idea is this: we should countenance plural quantifiers, and analogues of plural quantifiers for all types (just as we have countenanced analogues of first-order singular quantifiers for all types), and pluralities at any type must satisfy analogues of Extensionality, Persistence, and Inextensibility for the present notion of necessity. Notice that by adding Rigidity to Classicism we do not presuppose that Rigidity is necessary, nor even that it is determinate. The mere truth of Rigidity is sufficient for present purposes.

We will show in Classicism plus Rigidity that, if it is not possible for there to be any finite number of things, then the finite cardinality quantifiers, being zero in number, being one in number, and so on, play the role of natural numbers necessarily, and their structure is had necessarily. Interpreting arithmetical language as implicitly higher-order, so that quantification over numbers is in fact higher-order quantification over finite cardinality quantifiers, every arithmetical sentence will thereby state either $T$ or $\perp$; which is to say that there is no arithmetical contingency in the broadest possible sense. Arithmetical determinacy follows.

In what follows let $\sigma$ be any type, to be held fixed throughout ( $e$ is the most natural option, but nothing formal hangs on this). We require the following definitions to get started (as well as obvious translations of Definitions 1, 2, and 3 into $\mathcal{H}$ ).

Definition 5 (That many plus one). For there to be some number plus one of some things is for there to be that number of those things and then one left over. In

${ }^{6}$ See, for instance, Stalnaker 1976 and Lewis 1986. 
symbols:

$$
.^{+}:=\lambda Q^{(\sigma \rightarrow t) \rightarrow t} \lambda X^{\sigma \rightarrow t} . \exists y(X y \wedge Q(\lambda z . X z \wedge z \neq y)) .
$$

Definition 6 (Finite cardinality quantifier). The finite cardinality quantifiers are those properties of properties that have every property that (a) apply to being zero in number, and (b), apply to $Q^{+}$whenever they apply to a given cardinality quantifier $Q$. Intuitively, the finite cardinality quantifiers are generated from being zero in number by repeatedly applying the that many plus one operation. Formally:

$$
\text { FinCard }^{\sigma}:=\lambda Q . \forall Y\left(Y \exists_{0} \rightarrow \forall P^{(\sigma \rightarrow t) \rightarrow t}\left(Y P \rightarrow Y P^{+}\right) \rightarrow Y Q\right),
$$

where $\exists_{0}:=\lambda F .(\neg \exists x F x)$.

The finite cardinality quantifiers, we will find, play the role of natural numbers at the type of quantifiers, whenever it is possible for each finite number that there are at least that many things. In symbols:

$$
\forall Q(\text { FinCard } Q \rightarrow \diamond \exists X(Q X))
$$

Call this proposition Unboundedness. Few have the courage to deny Unboundedness, but it is instructive to see what hangs on this assumption. If Unboundedness fails, then one of the finite cardinality quantifiers necessarily applies to nothing. This is to say: starting with $\exists_{0}$, and repeatedly applying $\cdot^{+}$, you will eventually reach the property $\lambda X . \perp$. So, for instance, it might be that being two in number, $\lambda X .(\exists y z(y \neq z \wedge X y \wedge X z))$, which is $\exists_{0}^{++}$, is just $\lambda X . \perp$. Since $(\lambda X . \perp)^{+}=\lambda X . \perp$, it will turn out that applications of ${ }^{+}$eventually reach a fixed point. In that case, there will only be finitely many finite cardinality quantifiers, and they will not be suited to playing the role of natural numbers.

The result is a consequence of the following sequence of lemmas. We begin with two helpful results that are on the easier side:

Lemma 6 (C). Finite cardinality quantifiers are necessarily so.

$$
\forall Q(\text { FinCard } Q \rightarrow \square \text { FinCard } Q)
$$

Proof. That $\square$ FinCard $\left(\exists_{0}\right)$ is trivial, and by definition we have $\square \forall Q$ (FinCard $Q \rightarrow$ FinCard $Q^{+}$). Now suppose that $\square$ FinCard $Q$. Then $\square$ FinCard $Q^{+}$. So by induction, $\forall Q($ FinCard $Q \rightarrow \square$ FinCard $Q)$.

(It follows that Unboundedness is true if it is so much as possible: if it is possible that every FinCard is possibly instantiated, then every FinCard is possibly possibly instantiated by Lemma 6. But possible possibility in Classicism is just possibility, so every FinCard will be possibly instantiated.) 
Lemma 7 (C). Finite cardinality quantifiers are cardinality quantifiers, in the sense that equinumerous properties always have the same finite cardinality quantifiers in common:

$$
\forall P(\text { FinCard } P \rightarrow \square \forall X Y((X \approx Y) \rightarrow P X \rightarrow P Y)),
$$

where $\approx$ is the relation of equinumerosity; of there being a bijective functional relation between $X$ things and $Y$ things.

Proof. Clearly $\exists_{0}$ has this property. Now suppose that an arbitrary FinCard $P$ does. Then, if $P^{+} X$ holds and $\iota$ is a bijective relation between the $X \mathrm{~s}$ and $Y \mathrm{~s}$, then for some $x, P \lambda z \cdot(X z \wedge(x \neq z))$ and $\lambda x .(\iota y z \wedge(y \neq x))$ is a bijection between the $X$ things besides $x$ and the $Y$ things beside whatever $\iota$ maps $x$ to, $y$ say. Thus $P \lambda z .(Y z \wedge(y \neq z))$, so $P^{+} Y$. Applying Eq and Gen makes this reasoning universal and necessary.

The next few lemmas are more complex, but the general idea of repeatedly using proof by induction is the same.

Lemma 8 (C). If Unboundedness is true, ${ }^{+}$is injective on FinCard. Moreoever, its inverse can be defined explicitly as

$$
.^{-}:=\lambda Q . \lambda X . \forall P\left(\text { FinCard } P \rightarrow\left(P^{+}=Q\right) \rightarrow P X\right) .
$$

Proof. We first show that ${ }^{+}$is injective, which is to say that distinct FinCards have distinct successors. Suppose for reductio that, for some distinct FinCards $P$ and $Q$, $P^{+}=Q^{+}$, so that $\square \forall x\left(P^{+} x \leftrightarrow Q^{+} x\right)$. By Unboundedness, $\diamond \exists X\left(P^{+} X \wedge Q^{+} X\right)$, and by the definition of $\cdot^{+}$we have

$$
\square \forall X\left(\left(P^{+} X \wedge Q^{+} X\right) \rightarrow \exists y(P(\lambda z \cdot X z \wedge z \neq y) \wedge Q(\lambda z \cdot X z \wedge z \neq y))\right.
$$

It follows that if $P^{+}=Q^{+}$, then it is possible for $P$ and $Q$ to have an instance in common, which is to say that it is possible for there to be some things that have cardinality $P$ and cardinality $Q$ simultaneously.

We prove by induction that this is contradictory. That is, we will show by induction that for distinct FinCards $P$ and $Q$, it is impossible for $P$ and $Q$ to both apply to the same property. In the base case, we must show that $\exists_{0}$ does not possibly have any members in common with any other FinCard. This is so because each FinCard besides $\exists_{0}$ is of the form $P^{+}$for some FinCard $P$, and $P^{+}$can only ever apply to properties with at least one instance. This completes the base case for our inductive argument.

For the inductive step, suppose that an arbitrary FinCard $P$ is such that it does not possibly have any member in common with any other FinCard. We will show by 
induction that $P^{+}$therefore also has this property, completing the argument. For the base case, we already have that $P^{+}$does not possibly have any members in common with $\exists_{0}$. For the inductive step, let $Q$ be a FinCard that necessarily has no members in common with $P^{+}$. Suppose that $P^{+}$and $Q^{+}$possibly have a member in common:

$$
\left.\diamond \exists X\left(\exists y(Q \lambda z .(X z \wedge(y \neq z))) \wedge \exists y^{\prime}\left(P\left(\lambda z .\left(X z \wedge\left(y^{\prime} \neq z\right)\right)\right)\right)\right)\right)
$$

Since $\lambda z .(X z \wedge(y \neq z))$ and $\lambda z .\left(X z \wedge\left(y^{\prime} \neq z\right)\right)$ are always equinumerous, it follows by Lemma 7 that

$$
\diamond \exists X(\exists y(Q \lambda z .(X z \wedge(y \neq z)) \wedge P(\lambda z .(X z \wedge(y \neq z))))
$$

which is to say that possibly, $P$ and $Q$ have some member in common. Thus, $P=Q$, so $P^{+}=Q^{+}$as required.

We have thus established that ${ }^{+}$is injective given Unboundedness. It remains to show that $\cdot^{-}$is the inverse of ${ }^{+}$. To show that $Q=Q^{+-}$, use the definition of - and the fact that FinCards are necessarily FinCards to show that $Q$ and $Q^{+-}$are necessarily coextensive and are therefore identical. In the other direction, it is easy to check that $\exists_{0}^{-+}=\exists_{0}$, and where $Q^{+}$is a successor FinCard, use the fact that $Q=Q^{+-}$, so that $\left(Q^{+}\right)^{-+}=\left(Q^{+-}\right)^{+}=Q^{+}$as required.

We will show that the finite cardinality quantifiers form an inductive structure when ordered by

$$
\leq:=\lambda X Y . \text { FinCard } X \wedge \text { FinCard } Y \wedge \square \forall Z\left(Y Z \rightarrow \exists Z^{\prime} \forall x\left(Z^{\prime} x \rightarrow Z x\right) \wedge X Z^{\prime}\right) .
$$

(Also define $<:=\lambda X Y .(X \leq Y) \wedge(X \not \leq Y)$.)

Lemma 9 (C). If Unboundedness is true, then Order(FinCard, $\leq$ ).

Proof. Reflexivity is obvious. For transitivity suppose that $X \leq Y \leq Z$, then necessarily for any things that are $Z$ in number, there are some of those that are $Y$ in number, and for any things that are $Y$ in number, there are some of those that are $X$ in number. So $X \leq Z$. For antisymmetry, suppose $X \leq Y \leq X$. Then $\square \forall z z^{\prime}\left(X z \rightarrow Y z^{\prime} \rightarrow\left(z \approx z^{\prime}\right)\right)$, so $X=Y$ by the argument in the proof of Lemma 8 .

For totality of $\leq$, notice first that if $X \leq Y$, then $X<Y^{+}$, and if $X<Y$, then $X^{+} \leq Y$. By induction, then, $\exists_{0}$ can be compared with any FinCard by $\leq$. Furthermore, if $X$ can be compared with any FinCard by $\leq$, then $X^{+}$is greater than everything that $X$ is at least as great as, and at least as small as everthing $X$ is strictly smaller than. By Induction again, $\leq$ is total.

Lemma 10 (C). If Unboundedness is true, then Ind(FinCard, $\leq$ ), and the successor of any finite cardinality quantifier $Q$ is $Q^{+}$. 
Proof. Lemma 9 establishes Order(FinCard, $\leq$ ). That $\exists_{0} \leq X$ is evident, and induction holds by definition. It remains to show that $\operatorname{Suc} Q Q^{+}$for a given FinCard $Q$.

For this, notice that $Q \leq Q^{+}$by taking some things that are $Q^{+}$in number, and excluding one of them to get a subclass of things that are $Q$ in number. Thus $Q<Q^{+}$ because $Q \neq Q^{+}$(Lemma 8). Suppose for contradiction that $Q<P<Q^{+}$, then if any things are $Q^{+}$in number then there is a strict subclass that are $Y$ in number and not $Q^{+}$in number. But then those things besides at least two of them are $Q$ in number, a contradiction.

Lemma 11 (C). Let $U$ stand for Unboundedness. Then if FinCard $X$ and FinCard $Y$, then

$$
\square(U \rightarrow(X<Y)) \vee \square(U \rightarrow(X=Y)) \vee \square(U \rightarrow(Y<X))
$$

Proof. We have $\square((X<Y) \vee(X=Y) \vee(Y<X))$ for FinCard $X$ and $Y$ by necessitating Lemma 9. It suffices to show that if FinCard $X$ and FinCard $Y$, then if $X<Y$ then $\square(U \rightarrow(X<Y))$. Suppose for contradiction that $X$ be the first finite cardinality quantifier that is greater than another, $Y$, say, that it is possibly not greater than. Then $X^{-}$has this property unless $X^{-}=Y$. Since $X$ is least, $X^{-}$must be $Y$. But $\square\left(X^{-}<X\right)$ by induction.

Lemma 12 ( $\mathrm{C}+$ Rigidity). The rigid thing coextensive with FinCard is such that necessarily, if Unboundedness is true then it is coextensive with FinCard. Moreover, the rigid thing coextensive with $\leq$ is such that necessarily, if Unboundedness is true then it is coextensive with $\leq$.

Proof. Let $F$ be rigid and coextensive with FinCard. By Lemma 6, every $F$ thing is necessarily a FinCard, so it is necessary that every $F$ thing is a FinCard by the rigidity of $F$.

In the other direction: suppose for contradiction that possibly, Unboundedness is true while some FinCard is not $F$. There would then be a least such FinCard, $X$. $X$ would have to be distinct from $\exists_{0}$ because $\exists_{0}$ is necessarily $F$, so $X^{-}$would have to be a FinCard that is $F$, because $X$ was assumed to be least. So if it is possible for Unboundedness to be true while some FinCard is not $F$, it is possible for Unboundedness to be true while the successor of some $F$ thing is not $F$. By the rigidity of $F$, it's not the case that for every $F$, the successor of it is necessarily $F$. But $F$ is coextensive with FinCard and the successor of every FinCard is $F$ and so necessarily $F$, a contradiction.

Let $L$ be the rigid relation coextensive with $\leq$. Lemma 11 gives us $(X \leq Y) \rightarrow$ $\square(U \rightarrow(X \leq Y))$; and so $\square(U \rightarrow \forall X Y((X \leq Y) \rightarrow(L X Y)))$. In the other direction, 
use the rigidity of FinCard to show that if possibly $U$ is true and for some $X$ and $Y$, $L X Y$ but not $X \leq Y$, then for some FinCards $X$ and $Y$ it is possible that $L X Y$ but not $X \leq Y$, all while $U$ holds. But if it is possible that $X \leq Y$ is false while $U$ is true, then $X \leq Y$ is in fact false, so $L X Y$ is too.

These lemmas allow us to reason as in Section 4. Define the $S R$ - $\sigma$ formulae to be exactly like the $S R$-first-order formulae (Definition 4) but with $\sigma$ s replacing $e$ s in the types of all variables. To be precise:

Definition 7 ( $S R-\sigma$ formula/ $\sigma$-arithmetical formula). The class of $S R$ - $\sigma$ formulae is defined by recursion as follows:

- $x=y$ and $R x y$ are $S R-\sigma$ formulae for any variables $x$ and $y$ of type $\sigma$;

- When $\varphi$ and $\psi$ are $S R$ - $\sigma$ formulae, then so are: $\neg \varphi ; \varphi \rightarrow \psi$; and $\forall x(S x \rightarrow \varphi)$ for any variable $x$ of type $\sigma$.

An $S R-\sigma$ sentence is an $S R-\sigma$ formula with no free variables besides $S$ and $R$.

Let a $\sigma$-arithmetical formula be an $S R$ - $\sigma$ formula but with FinCard replacing $S$ and $\leq$ replacing $R$, and define $\sigma$-arithmetical sentence analogously.

Theorem 13 (C + Rigidity). Let U stand for Unboundedness, and let $\varphi$ be a $\sigma$ arithmetical sentence. Then

$$
\square(U \rightarrow \varphi) \vee \square(U \rightarrow \neg \varphi) .
$$

Proof. Lemma 11 says that distinct finite cardinality quantifiers are necessarily distinct while Unboundedness holds, and Lemma 12 that the finite cardinality quantifiers with their defined ordering behave rigidly when Unboundedness is true. Repeating the proof of Lemma 3 in Classicism plus Rigidity yields the desired result.

Corollary 14 (C + Rigidity). Let $U$ stand for Unboundedness, and for an arbitary type $\tau$ let Ind $d_{\tau}$ of type $(\tau \rightarrow t) \rightarrow(\tau \rightarrow \tau \rightarrow t) \rightarrow t$ be the higher-order property of being $a \tau \rightarrow t$ thing and $a \tau \rightarrow \tau \rightarrow t$ thing that form an inductive structure (Definition 2). Then

$$
\square\left(U \rightarrow \forall S R\left(\operatorname{Ind}_{\tau} S R \rightarrow \varphi\right)\right) \vee \square\left(U \rightarrow \forall S R\left(I n d_{\tau} S R \rightarrow \neg \varphi\right)\right),
$$

where $\varphi$ is an SR-first-order sentence.

Proof. Repeating the proof of Theorem 2 in Classicism, we have that necessarily, if Unboundedness is true then every inductive structure is isomorphic to the finite cardinality quantifiers. Thus, every $S R-\tau$ sentence is true if and only if the corresponding $\sigma$-arithmetical sentence is true. 
The upshot of Theorem 13 is that, given Unboundedness, there can be no modal variation in the propositions expressed by $\sigma$-arithmetical sentences. The $\sigma$ arithmetical sentence stating the analogue of the Goldbach conjecture, for instance, expresses either $T$ or $\perp$. Corollary 14 shows that there can also be no variation in the first-order satisfaction properties of inductive structures generally. If it is possible that one satisfies the Goldbach conjecture while Unboundedness is true, then necessarily all do. It follows that, given Unboundedness, general worries about the modal strength of distinctness facts do not affect the core argument for the determinacy of arithmetic.

For the nominalist, the more important point is the observation that given Unboundedness, the finite cardinality quantifiers form a modally rigid and necessarily inductive structure. So the denial of the existence of an inductive structure made of individuals does not allow for indeterminacy in what such structures would be like if there were any. Another consequence is that nominalist-friendly paraphrase schemes for arithmetical language are guaranteed to be modally well-behaved in a way that is probably the best sort of "arithmetical determinacy" that a nominalist could hope for. The idea is that the nominalist may paraphrase apparent reference to numbers and numberhood in a higher-order language, so that she treats the apparent predicate "is a number" as code for a higher-order operator akin to "is a finite cardinality quantifier," the apparent name "zero" as code for the quantifier-expression " $\exists_{0}$," and quantification over "numbers" as higher-order quantification over finite cardinality quantifiers. The propositions expressed by such higher-order sentences will all be either necessitated by or incompatible with Unboundedness. Since even nominalists can think that Unboundedness is determinately true, there is some good sense in which they can agree that arithmetic is both true and determinate. ${ }^{7}$

\section{References}

Bacon, A. (2018). "The Broadest Necessity". In: Journal of Philosophical Logic 47.5, pp. 733-783.

Bacon, A. and C. Dorr (forthcoming). "Classicism”. In: Higher-Order Metaphysics. Ed. by P. Fritz and N. K. Jones. OUP.

Button, T. and S. Walsh (2016). "Structure and Categoricity: Determinacy of Reference and Truth Value in the Philosophy of Mathematics". In: Philosophia Mathematica 24.3, pp. 283-307.

${ }^{7}$ This paper has greatly benefited from discussion with Cian Dorr and Jeff Russell, and comments from Jeremy Goodman and two anonymous referees for this journal. Special thanks are due to Andrew Bacon, who provided indispensable philosophical and logical advice from start to finish. 
Church, A. (1940). "A Formulation of the Simple Theory of Types". In: Journal of Symbolic Logic 5.2, pp. 56-68.

Dedekind, R. (1893). Was Sind Und Was Sollen Die Zahlen? F. Vieweg.

Evans, G. (1978). "Can There Be Vague Objects?” In: Analysis 38.4, p. 208.

Feferman, S. et al. (1999). “Does Mathematics Need New Axioms?” In: Bulletin of Symbolic Logic 6, pp. 401-413.

Field, H. (1998). "Which Undecidable Mathematical Sentences Have Determinate Truth Values". In: Truth in Mathematics. Ed. by H. G. Dales and G. Oliveri. Oxford University Press, Usa, pp. 291-310.

Frege, G. (1884). Grundlagen der Arithmetik. Breslau: Wilhelm Koebner.

Hamkins, J. D. (2012). “The Set-Theoretic Multiverse”. In: Review of Symbolic Logic 5.3, pp. 416-449.

- (2015). "Is the Dream Solution of the Continuum Hypothesis Attainable?" In: Notre Dame Journal of Formal Logic 56.1, pp. 135-145.

Lewis, D. (1984). "Putnam's Paradox". In: Australasian Journal of Philosophy 62.3, pp. 221-236.

- (1986). On the Plurality of Worlds. Wiley-Blackwell.

Linnebo, Ø. (2013). “The Potential Hierarchy of Sets”. In: Review of Symbolic Logic 6.2, pp. 205-228.

Martin, D. (2001). "Multiple Universes of Sets and Indeterminate Truth Values". In: Topoi 20.1, pp. 5-16.

McGee, V. (1997). "How We Learn Mathematical Language". In: Philosophical Review 106.1, pp. 35-68.

Parsons, C. (2007). Mathematical Thought and its Objects. Cambridge University Press.

Quine, W. V. O. (1970). Philosophy of Logic. Harvard University Press.

Stalnaker, R. (1976). "Propositions". In: Issues in the Philosophy of Language: Proceedings of the 1972 Colloquium in Philosophy. Ed. by A. F. MacKay and D. D. Merrill. New Haven and London: Yale University Press, pp. 79-91.

Warren, J. and D. Waxman (2020). "A Metasemantic Challenge for Mathematical Determinacy”. In: Synthese, pp. 1-19. 